\title{
New Energy Vehicles Adoption in the Beijing-Tianjin-Hebei Region
}

\author{
Xue Bai ${ }^{1, *}$, Haojie Sun ${ }^{2}$ \\ ${ }^{1}$ School of Economics \& Management, The Open University of China, 75 Fuxing Road, Haidian District, Beijing 100039, China \\ ${ }^{2}$ School of Management and Economics, Beijing Institute of Technology, 5 South Zhongguancun Street, Beijing 100081, China
}

\begin{abstract}
Incentive policies play a key role in the development of new energy vehicles (NEVs) in China. With the analysis of 89 national and Beijing-Tianjin-Hebei (Jing-Jin-Ji) regional NEV policies from January 2006 to June 2018, this paper presents an extended category of subsidy policies. It shows that during the NEV adoption process, more and more emphasis has been laid on indirect subsidies. Although local NEV adoption is uneven and some cities lag behind the national pace to some extent, the regional coordination and integration has potential to boost the popularization of NEVs in an environmental and sustainable way. These findings help to build comprehensive policy systems for the maturity of NEV adoption in the future.
\end{abstract}

\section{Introduction}

New energy vehicles (NEVs) are of critical importance for China as we have faced with tense energy supply, poor air quality, transforming and upgrading the automotive industry [1-3]. In 2017, the NEV production and sales volume climbed to 0.794 and 0.777 million, respectively, attaining an year-on-year growth of $53.58 \%$ and $53.25 \%$. It is apparent that incentive policies have played an important role in achieving these impressive development. NEV subsidies as well as demonstration projects have brought positive effect on reaching adoption targets.

As stated in "the Energy-saving and New Energy Vehicle Industry Development Plan (2012-2020)", NEVs are fully or mainly driven by new energy, including battery electric vehicles (BEVs), plug-in hybrid electric vehicles (PHEVs) and fuel cell vehicles (FCVs). To further speed up the large-scale NEV adoption, the central government activated the pilot project participated by two batches of 88 cities and metropolis areas in November 2013 and January 2014, as shown in Fig. 1. Among them, the Beijing-Tianjin-Hebei region, also known as the Jing-Jin-Ji region, consists of two municipalities (Beijing and Tianjin) and 11 cities (Shijiazhuang, Baoding, Cangzhou, Chengde, Handan, Hengshui, Langfang, Qinhuangdao, Tangshan, Xingtai, Zhangjiakou) in Hebei province. Being one of the national strategies, the coordinated development of Jing-Jin-Ji region is promising to have a significant impact on the NEV adoption. The regional implementation roadmap and subsidy policy system can provide us with favorable references on the promotion of NEVs nationwide.

This research focuses on the timeline-based subsidy policies analysis which differs from [4-7]. It also differs from the traditional method which clusters policies into the supply, demand or environment group [8,9]. The drawback of $[8,9]$ is that many NEV incentive policies contain both supply end and demand end measures, and they cannot be classified into one single group strictly. Instead, this paper proposes an extended category of NEV subsidy policies and related policy systems. By illustrating the interrelation among different NEV policies promulgated by different levels of governments, it helps to reveal how they are implemented with detailed tasks and how they function as a whole to promote the NEV market adoption. The remainder of the paper is organized as below: Section 2 presents the extended category of NEV subsidy policies. The national and Jing-Jin-Ji regional NEV policy systems are analyzed in section 3 and section 4 , respectively. Section 5 discusses the findings and conclusions.

\section{Methodologies}

We collected $89 \mathrm{NEV}$ policies promulgated from January 2006 to June 2018 from government websites. Ten of them were newly published in 2017 and 2018. 41 policies were released by the central government, with 8 regional policies and 40 local policies. 


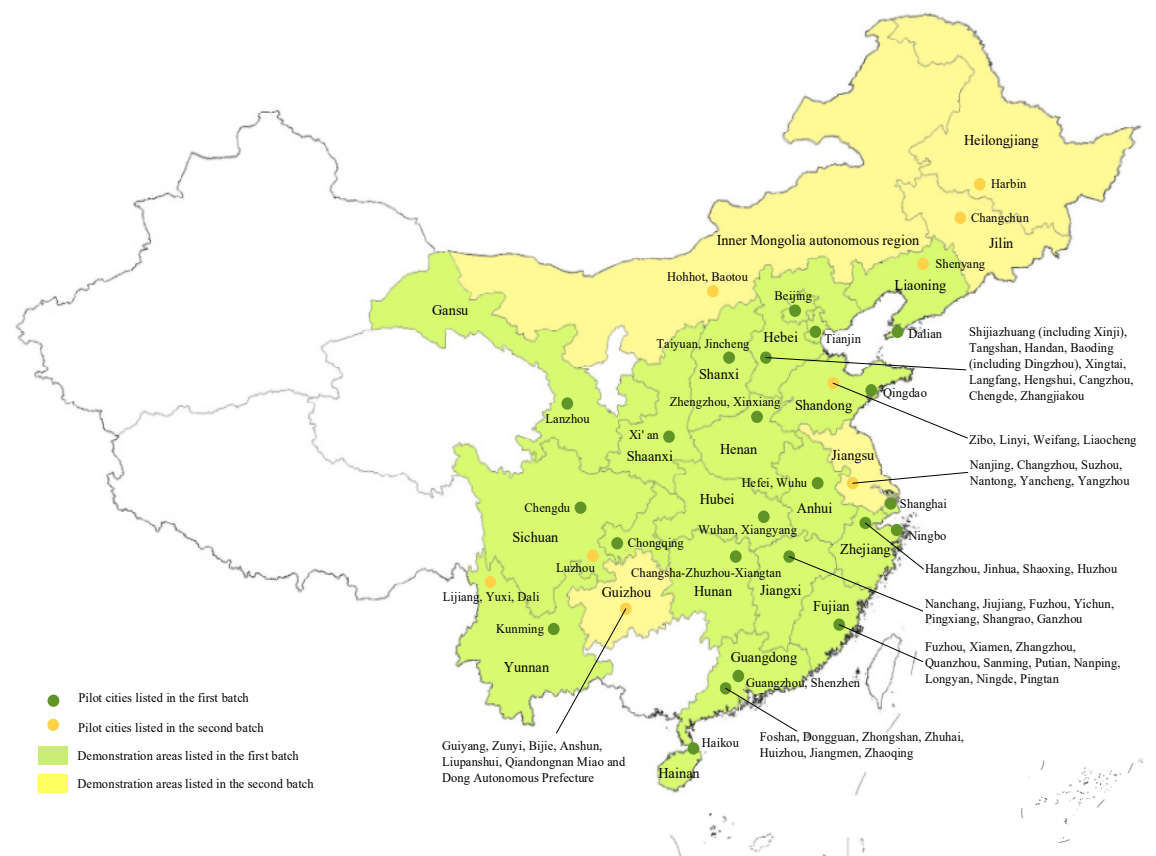

Fig. 1. NEV demonstration areas in China

Based on the category of NEV subsidies used in [10], this paper analyzes both direct and indirect NEV subsidy policies. As shown in Fig. 2, those indirect subsidy policies are further classified into six groups. Since governments at different levels have paid great attention to air pollution prevention and control, specific policies focusing on vehicle emissions reduction should be classified into a new group.

As to the irreplaceable role played by national incentive policies, every demonstration program with valid period is showed by one rectangle with the corresponding color and length in the upper part. It helps to disclose major missions during different NEV development stages. National NEV policy associated with a certain adoption project is listed in the lower part according to its release time. When taking local NEV policies into consideration, there is necessity to analyze pilot programs launched in Beijing, Tianjin, Hebei, and the Jing-Jin-Ji region, respectively.

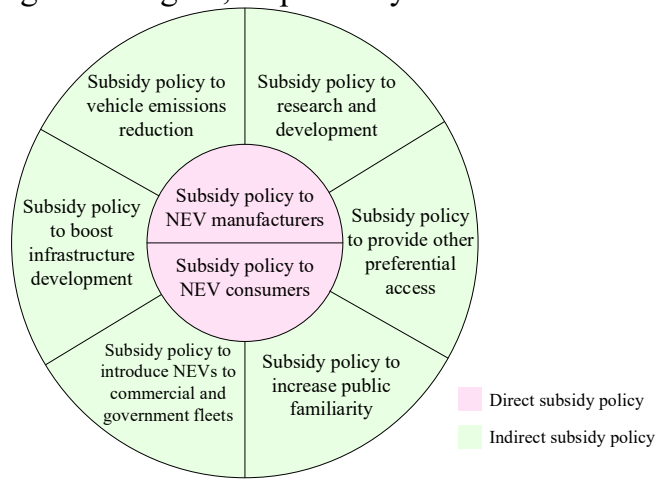

Fig. 2. Category of NEV subsidy policies

\section{National NEV policies}

As the strategic emerging industry, the development of new energy vehicles has been encouraged by Chinese government especially since 2006 [11] with the aim of reducing air pollution, conserving the fuel oil energy and upgrading the automotive industry. Given that China has become the world's largest producer and consumer of automobiles, both development guidelines and adoption strategies have potential to make the positive effect on the popularization and application of NEVs worldwide [12].

As shown in Fig. 3, "the Access Management Rules for New Energy Vehicle Production" was promulgated in October 2007. It first declared the standard norms of new energy vehicle industry. With preferential access rules announced by the central government, the "Ten Cities, Each with One Thousand NEVs" Project for public adoption and the pilot program for subsidizing private purchasers were first launched in 2009 and 2010, respectively. Preferential tax policies were issued to create favorable circumstances facilitating industry development and to raise the public awareness of environmental protection gradually.

For further implementation of energy conservation and emissions reduction, "the Energy-saving and New Energy Vehicle Industry Development Plan (2012-2020)" was promulgated as the leading guideline. It gave priority to the development of BEVs and PHEVs, and set the cumulative production and sales volume to reach 500 thousand by the end of 2015. To make key breakthroughs in battery packs, energy management systems and other advanced techniques, "the Twelfth Five-Year Special Plan of Electric Vehicle Technology Development" and "the Circular on Launching the Technological Innovation Project of New Energy Automotive Industry" established the "three-step" NEV development roadmap during 2012-2020. In addition to mitigate the climate change and achieve the sustainable development of economy and society, "the Air Pollution Prevention and Control Action Plan" with detailed work arrangement declared that NEVs should be widely applied in both public transport and government fleet. 


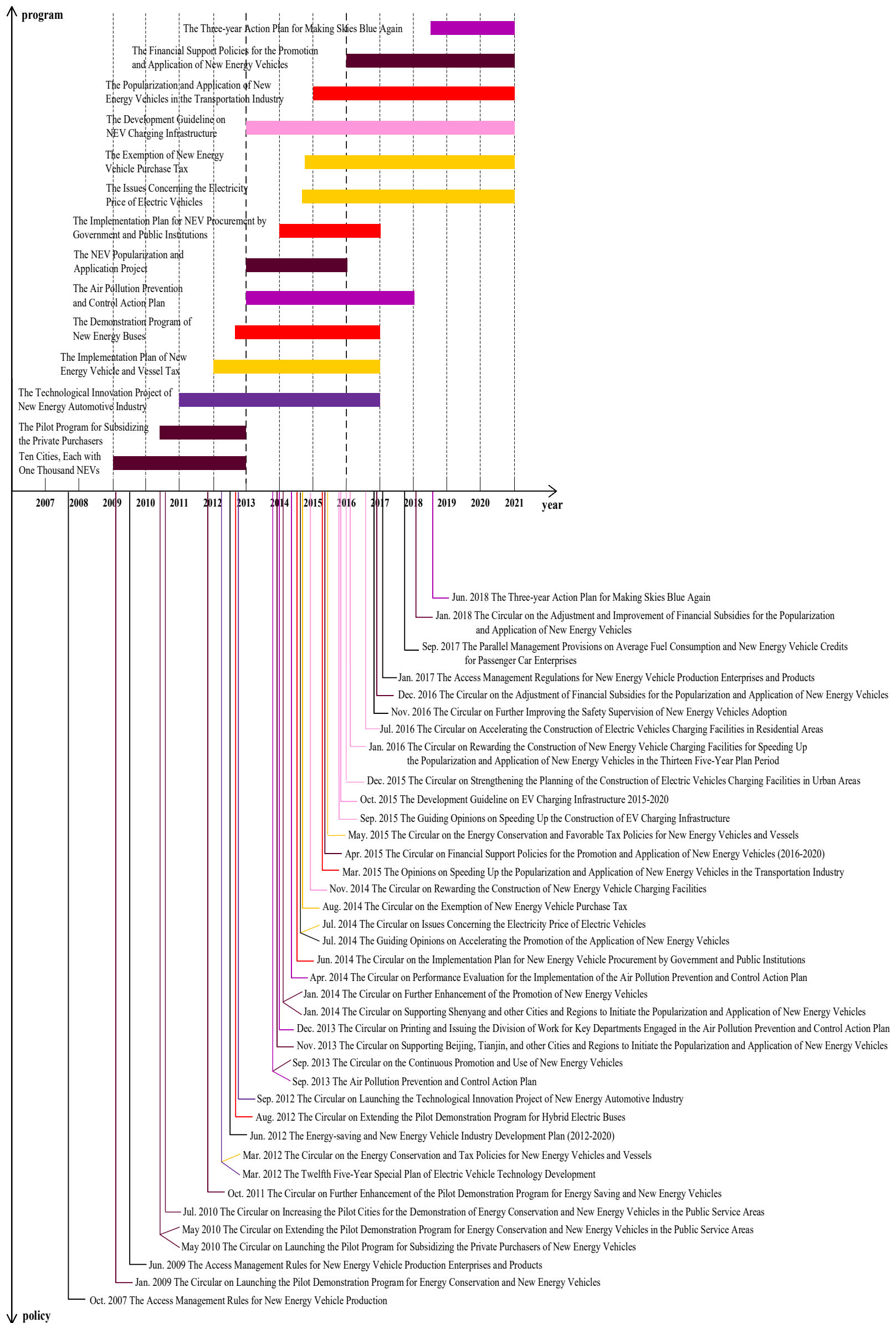

Fig. 3. Category of national NEV policies 
In 2013, the new NEV pilot project was started with 88 cities and areas. The central government decided to phase out subsidies gradually since 2013 with the aim of forcing NEV industry to speed up R\&D breakthroughs and make products sufficiently competitive in the sales market. "The Circular on the Implementation Plan for New Energy Vehicle Procurement by Government and Public Institutions" and "the Opinions on Speeding Up the Popularization and Application of New Energy Vehicles in the Transportation Industry" stated to expand the adoption by further introducing NEVs to taxi service, postal service, logistics and urban tourism areas.

In July 2014, "the Guiding Opinions on Accelerating the Promotion of the Application of New Energy Vehicles" was issued to accelerate the improvement of policy systems, strengthen the construction of charging facilities, and implement the elimination of local protectionism determinedly. Following the overall planning, "the Guiding Opinions on Speeding Up the Construction of EV Charging Infrastructure", "the Development Guideline on EV Charging Infrastructure 2015-2020", and detailed management provisions were released to build efficient and intelligent charging infrastructure systems to meet the charging demand of more than 5 million electric vehicles by the end of 2020 . Attractive subsidies intend to be assigned to local governments with large adoption scale, with the maximum amount of 120 million RMB in 2016 and 200 million RMB in 2020.

As stated in "the Circular on Financial Support Policies for the Promotion and Application of New Energy Vehicles (2016-2020)", the NEV adoption needs to be launched nationwide during the period of the Thirteenth Five-Year Plan. Financial subsidies phasing out annually contributes to further pushing NEV industry to enhance the core competitiveness. In addition, revised preferential policies regarding the NEV purchase tax and charging price were published to increase the pubic familiarity during 2016-2020. In 2017, "The Parallel Management Provisions on Average Fuel Consumption and New Energy Vehicle Credits for Passenger Car Enterprises" proposed the new accounting method to clarify how the positive credits of NEVs deduct the corresponding negative credits of average fuel consumption within or among passenger car enterprise(s). These subsidy policies, both direct and indirect ones, have played significant roles in NEV adoption, especially in the pilot demonstration of the Jing-Jin-Ji region.

\section{Jing-Jin-Ji regional NEV policies}

As the capital city and international metropolis, Beijing took the lead in promoting NEVs for public services and private consumers since 2010 and adopted 67803 NEVs in 2017. Together with Beijing and 10 cities in Hebei province, Tianjin was listed in the first batch of 28 cities and areas to formally initiate the NEV demonstration program in 2013. It achieved the adoption of $39192 \mathrm{NEVs}$ in 2017. Qinhuangdao, another Hebei city, was included in the work plan for NEV promotion in public service areas since 2014. In the past year, $18925 \mathrm{NEVs}$ were adopted in Hebei province.

The Jing-Jin-Ji regional NEV policy system was shown in Fig. 4. By the end of 2012, Beijing, Tianjin and Hebei had released general incentive measures. Sufficient funds were required to be reserved for local incentive subsidies. Following the national "Air Pollution Prevention and Control Action Plan", the Jing-Jin-Ji regional, Hebei provincial, Beijing and Tianjin municipal yearly work plans were promulgated to pioneer the NEV adoption in public transit, government fleet, taxi and sanitation service, etc.

During 2013-2015, the regional policy system was getting improved significantly. Guiding policies were promulgated by Beijing, Tianjin, and Hebei governments, followed by a series of detailed subsidy rules and management regulations. In 2014, "the Beijing Electric Vehicle Promotion Action Plan (2014-2017)" announced that 170000 plates of electric passenger cars should be assigned to private purchasers in the next four years. Consumers were able to obtain both national and local subsidies if the total were no more than $60 \%$ of the corresponding sales price. "Tianjin New Energy Vehicle Pilot Demonstration Program (2013-2015)" declared to adopt 120 thousand NEVs by the end of 2015. National and municipal subsidies were allocated at the ratio of $1: 1$. "The Opinions on Accelerating the Development and Promotion of New Energy Vehicles in Hebei" was published on July 3, 2014. Provincial subsidies were set the same as the national for NEV adoption in public service sectors, and the proportion changed into $0.5: 1$ when it came to other application areas. As for the coordinated demonstration program in the Jing-Jin-Ji region, the Ministry of Industry and Information Technology joint with other ministries announced the general adoption target of 20222 NEVs during 2014-2015. They also designated yearly tasks to Beijing, Tianjin, and Hebei, respectively.

To improve the continuity and fullness of incentive policy systems during the period of the Thirteenth Five-Year Plan, Beijing updated financial subsidies of all-electric buses and special vehicles. Tianjin prioritized to expand the production scale, enhance the innovation ability, and promote wider NEV adoption. In 2015, Hebei promulgated "the Thirteenth Five-Year Plan for Hebei New Energy Automotive Industry", aiming to build one or two NEV manufacturers with international influence and establish a group of NEV industrial bases. To improve the efficiency in the uses of financial funds, all-electric city buses could obtain certain amount of provincial and municipal subsidies, while other types of NEVs were no longer subsidized since July 11, 2016.

The long-term planning certainly requires joint efforts of all 13 cities in the Jing-Jin-Ji region and the Beijing-Tianjin-Hebei integration has witnessed a great breakthrough since 2015. "The Guideline for the Coordinated Development of the Beijing-Tianjin-Hebei Region" proposed to further implement the NEV adoption by connecting the dead-end highways and developing charging infrastructure along the intercity highways. It is also essential to set up the integrated network system for increased supervision and inspection. 


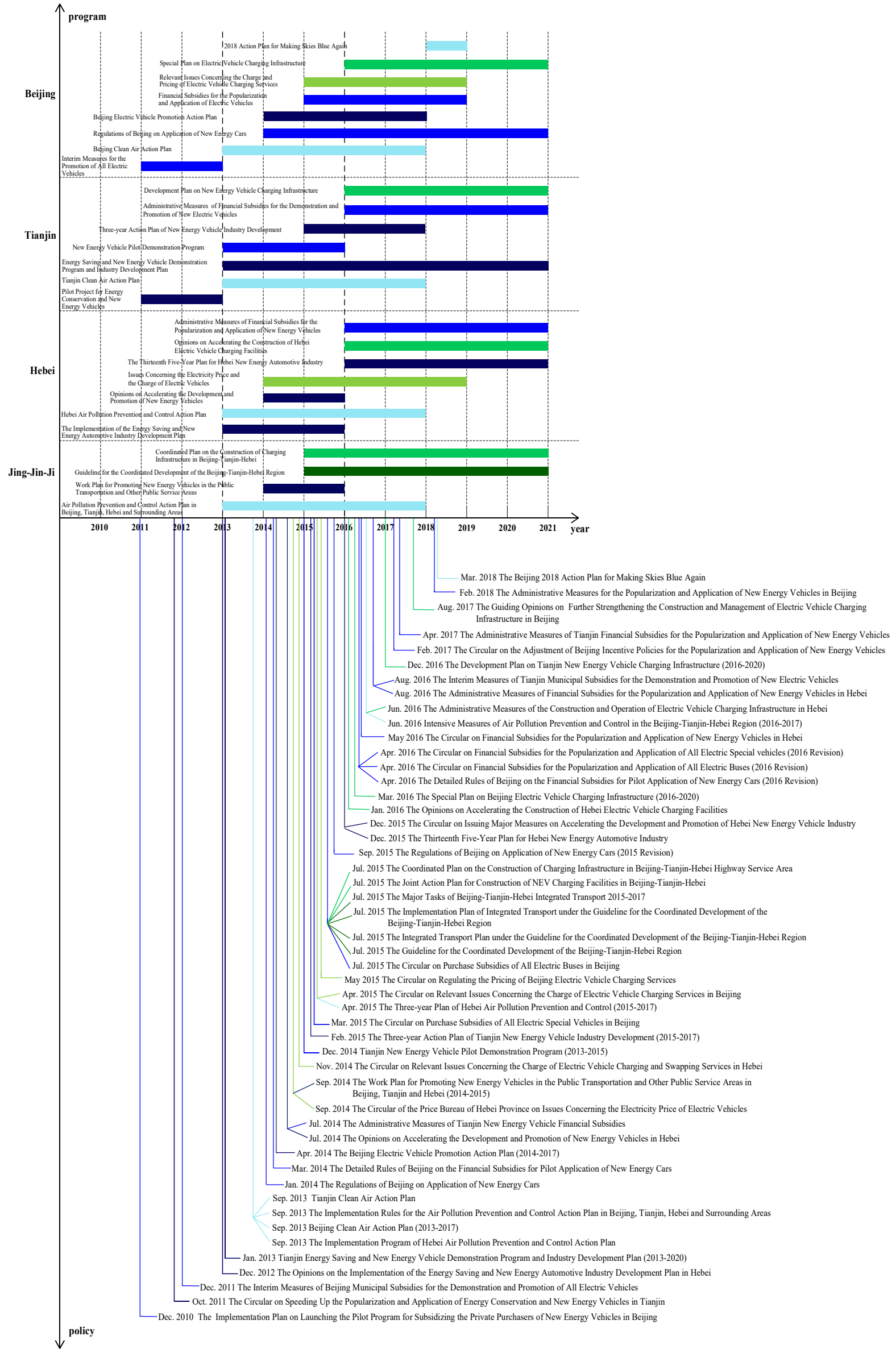

Fig. 4. Category of Jing-Jin-Ji NEV policies 
With supporting policies targeting at charging services and infrastructure construction, the NEV policy systems of Beijing, Tianjin and Hebei are getting well-functioning, as shown in Fig. 4. Early in 2015, Beijing activated "the Circular on Relevant Issues Concerning the Charge of Electric Vehicle Charging Services in Beijing" and "the Circular on Regulating the Pricing of Beijing Electric Vehicle Charging Services". Hebei specified the electricity price as well as the charge of charging and swapping services. Quickly responded by Beijing, Tianjin, and Hebei governments, they announced to boost the construction of "Internet + Charging Infrastructure". It will help to improve the convenience of charging services by integrating resources from different network platforms. From 2017 to June 2018, Beijing and Tianjin updated administrative measures for large-scale NEVs adoption, launching the adjustment dynamically in accordance with national subsidy policies.

\section{Discussion and conclusions}

Both the central and local governments have made efforts to promote the NEV adoption by incentive polices with different emphasis. Demonstration programs launched in different development stages have made consumers familiarized with both the public and private applications of NEVs with energy-efficient advantages and environmental impression. This paper used $89 \mathrm{NEV}$ policies, which promulgated at national, Jing-Jin-Ji regional, Hebei provincial and Beijing, Tianjin municipal levels from January 2006 to June 2018, to build an extended category of NEV subsidy policies to uncover their impacts upon the rapid NEV adoption in China.

As can be seen from the national policy system, national policies were mainly in the form of direct subsidies before 2013. The NEV demonstration programs for public sector and private purchase were initiated in pilot cities. During 2013-2015, more attention was paid to the role which NEV adoption played in the improvement of air quality. Consumers were encouraged to choose NEVs with preferential tax policies. To promote the sustainable development of new energy vehicles nationwide, the central government has attached more importance to the construction of charging service network since 2015. NEV technical standards and management regulations are promising to make impressive progress from 2016 to 2020 .

Local incentive policies were mainly promulgated as guidelines in the initial development stage before 2014 . Both direct and indirect subsidy policies were released intensively since 2014 and updated continually in the following years. Facing that the NEV adoption is uneven in the Jing-Jin-Ji region, Beijing owns the relatively comprehensive NEV policy system and favorable foundation for charging facilities construction. Work plans for the integrated transport have been jointly signed by Beijing, Tianjin, and Hebei governments. NEV policy systems with consistency and correlation are crucial for the NEV future development in China.
This study is supported by the Key Research Program of Beijing Social Science Foundation (No.15JDJGA021), the International Clean Energy Talents Program of China Scholarship Council (Liujinfa[2017]5047).

\section{References}

1. X. Yuan, X. Liu, J. Zuo. The development of new energy vehicles for a sustainable future: A review. Renewable and Sustainable Energy Reviews, 42, 298-305 (2015).

2. X. Zhang, X. Bai. Incentive policies from 2006 to 2016 and new energy vehicle adoption in 2010-2020 in China. Renewable and Sustainable Energy Reviews, 70, 24-43 (2017).

3. X. Zhang, Y. Liang, E. Yu, R. Rao, J. Xie. Review of electric vehicle policies in China: Content summary and effect analysis. Renewable and Sustainable Energy Reviews, 70, 698-714 (2017).

4. C. Lu, K. Rong, J. You, Y. Shi. Business ecosystem and stakeholders' role transformation: Evidence from Chinese emerging electric vehicle industry. Expert Systems with Applications, 41(10), 4579-4595 (2014).

5. J. Zheng, S. Mehndiratta, J. Y. Guo, Z. Liu. Strategic policies and demonstration program of electric vehicle in China. Transport Policy, 19, 17-25 (2012).

6. W. Li, R. Long, H. Chen. Consumers' evaluation of national new energy vehicle policy in China: An analysis based on a four paradigm model. Energy Policy, 99, 33-41 (2016).

7. Y. Liu, A. Kokko. Who does what in China's new energy vehicle industry?. Energy Policy, 57, 21-29 (2013).

8. C. Y. Huang, J. Z. Shyu, G. H. Tzeng. Reconfiguring the innovation policy portfolios for Taiwan's SIP Mall industry. Technovation, 27(12), 744-765 (2007).

9. R. Rothwell, W. Zegveld. Reindustrialization and Technology. Essex, Longman (1985).

10. X. Zhang. Reference-dependent electric vehicle production strategy considering subsidies and consumer trade-offs. Energy Policy, 67, 422-430 (2014).

11. The State Council. The Eleventh Five-Year Plan. Beijing (2006).

12. J. P. Helveston, Y. Liu, E. M. Feit, E. Fuchs, E. Klampfl, J. J. Michalek. Will subsidies drive electric vehicle adoption? Measuring consumer preferences in the U.S. and China. Transportation Research Part A: Policy and Practice, 73, 96-112 (2015).

\section{Acknowledgment}

